\title{
Attributed Favourable Relevance and Argument Evaluation ${ }^{I}$
}

\section{Derek Allen}

University of Toronto

\begin{abstract}
I criticize a case made by George Bowles for a certain theory pertaining to the evaluation of arguments on which the (degree of) attributed favourable relevance of an argument's premises to its conclusion is relevant to its evaluation, but nevertheless argue that such favourable relevance is indeed relevant to an argument's evaluation.
\end{abstract}

Résumé: Je critique un propos qui a fait George Bowles en appui d'une certaine théorie à propos de l'évaluation des arguments dans laquelle le degré de la relevance favorable des premisses de l'argument sur sa conclusion se bome sur son évaluation; mais j'argue, néanmoins, que de telle relevance favorable se borne à l'évaluation des arguments.

Keywords: argument; actual (attributed) favourable relevance (conclusive or inconclusive) of an argument's premises to its conclusion; George Bowles; good argumentative reasoning; premise-conclusion relation; proposition associated with an argument; reasoning; relevance proposition.

Is the attribution to an argument's premises of favourable relevance to its conclusion relevant to its evaluation? In an article published in this journal George Bowles has defended a theory which would say that it is - a theory of when an argument is good with respect to the relation between its premises and conclusion (Bowles 1991, cited hereafter as ' $E A$ '). I examine his defense of this theory, and find it unconvincing. Then, after considering but not endorsing a revised version of the theory, I connect attributed favourable relevance to argument evaluation via what I argue is a necessary condition of good argumentative reasoning.

First, then, Bowles's discussion. He assumes that one proposition, ' $p$ ', is either relevant or irrelevant to another proposition, ' $q$ ', "in what might be called the 'probative sense" "(EA:1). If ' $p$ ' is in this sense relevant to ' $q$ ', "it is so either favorably or unfavorably" (EA:1). "If " $p$ ' is favorably or unfavorably relevant to ' $q$ ', then it is so either conclusively or inconclusively" (EA:1). If ' $p$ ' entails ' $q$ ', then ' $p$ ' is conclusively favourably relevant to ' $q$ ', while if ' $p$ ' makes ' $q$ ' only probable, then ' $p$ ' is only inconclusively favourably relevant to ' $q$ ' (EA:1).

Relevance and irrelevance "may be actual, attributed, or both" (EA:1). For exam$\mathrm{ple}$, in a text of the form 'the fact that $p$ makes it certain that $q$ ', conclusive favourable relevance to ' $q$ ' is attributed to 'p' and actually belongs to ' $p$ ' if 'p' entails ' $q$ '. A text of

(ㅇ Informal Logic Vol. 18, Nos. $2 \& 3$ (1996): pp. 183-199. 
that form expresses an argument. By an argument Bowles understands "the smallest unit of reasoning, consisting of at least one premise and exactly one conclusion" (EA:1), and he holds that "[i]n an argument, to some propositions (namely, the premises) is attributed favorable relevance to another (the conclusion)" (EA:1). ${ }^{2} \mathrm{He}$ further holds, as an assumption, that a text of the form 'the fact that $p$ makes it certain that q' expresses an argument whose premise is ' $p$ ' and whose conclusion is ' $q$ '-not ' $q$ is certain'; similarly, a text of the form 'the fact that $p$ makes it probable that q', expresses an argument whose premise is ' $p$ ' and whose conclusion is ' $q$ ' - not ' $q$ is probable' (EA:2).

With these preliminaries out of the way, Bowles proceeds to examine six theories that have been given in answer to the question "[u]nder exactly what conditions is an argument good [with respect to the relation between its premises and conclusion], and under what conditions is it bad" with respect to that relation (EA:2). The first four theories all say that whether an argument is good or bad with respect to the relation between its premises and conclusion "is determined only by the actual, not even in part by the attributed, degree of favorable relevance of its premises to its conclusion" (EA:2), while the remaining two theories say that whether an argument is good or bad with respect to the relation between its premises and conclusion "is determined partly by the actual, and partly by the attributed, degree of favorable relevance" of its premises to its conclusion (EA:2).

Bowles finds the first five theories to be defective. I will not state these theories, or report Bowles's reasons for finding them defective, because my interest is in Theory 6 , which Bowles considers the best of the lot. ${ }^{3}$ This theory says that an argument is good with respect to the relation between its premises and conclusion "if the attributed and actual degrees of favorable relevance of its premises to its conclusion agree" (EA:9); otherwise the argument is bad with respect to that relation. "The attributed and actual degrees of favorable relevance of premises to a conclusion agree", according to Bowles, "if the actual degree either coincides with, or else falls entirely within the limits of, the attributed" (EA:9). Thus a text of the form "the fact that $p$ makes it certain that q' expresses an argument that is good with respect to the relation between its premise and conclusion just in case ' $q$ ' is certain given ' $p$ ', for in this case, and only in this case, the attributed and actual degrees of favourable relevance of the premise, ' $p$ ', to the conclusion, ' $q$ ', agree.

In reply to an objection to Theory 6 , Bowles remarks that "[w]ith each argument is associated a proposition that says, at the minimum, that its premises are favorably relevant to its conclusion. Sometimes, and perhaps always, this proposition is more detailed, saying, for example, either that the premises are conclusively favorably relevant to the conclusion or that they are to some degree inconclusively favorably relevant to it" (EA:10-11). According to Theory 6, "whether an argument is good or bad [with respect to the relation between its premises and conclusion] depends entirely on whether the proposition associated with that argument is true or false" (EA:11). Bowles believes that there is more to be said for this view than against it, ${ }^{4}$ and he therefore affirms that the proposition associated with an argument may "be thought of 
as specifying the conditions under which the argument is good" (EA:11) with respect to the relation between its premises and conclusion.

I turn now to four objections to Theory 6 , the first two of which are stated (and replied to) by Bowles. ${ }^{5}$

Objection 1: Contrary to a presupposition of Theory 6 , the logical evaluation of an argument does not involve ascertaining whether it has the features it ought to have. Rather, it involves "ascertaining whether certain logical relations really hold between the argument's premises and its conclusion" (EA:7). To say, for instance, that an argument is valid is only to say that its premises entail its conclusion. It is not to say that the argument is good, "actually possessing features that it ought to possess" (EA:7). Thus,

the logical evaluation of an argument is independent of all features that belong to the argument's context rather than to the argument itself. One such feature is the (degree of attributed favorable relevance of its premises to its conclusion. So, the logical evaluation of an argument is independent of the (degree of) attributed favorable relevance of its premises to its conclusion. Such favorable relevance may tell us something about the arguer's state of mind, and it may therefore pertain to the evaluation of the arguer, but it has nothing to do with the evaluation of his argument (EA:7).

Bowles's reply:

The logical evaluation of an argument with respect to the relation between its premises and conclusion ... consists in judging the argument's worth. The meanings of the terms of logical evaluation, such as 'valid' and 'invalid', include the notions of 'good' or 'bad'. For it would be self-contradictory logically to evaluate an argument as valid (strong, cogent, etc.) and yet to say that the same argument is in no way good; and it would be self-contradictory logically to evaluate an argument as invalid (weak, fallacious, etc.) and yet to say that the same argument is in no way bad. Consequently, since the logical evaluation of an argument consists in judging whether it is valid (strong, cogent, etc.) or invalid (weak, fallacious, etc.), that evaluation involves at least judging whether the argument is in some way good or bad. And to judge this is to judge whether it actually has the features that it ought to have. So, whether or not it has any bearing on the evaluation of the arguer, such a contextual feature as the attributed (degree of) favorable relevance of the premises to the conclusion may have a bearing on an argument's evaluation (EA:7).

Bowles thinks, then, that the logical evaluation of an argument "involves at least judging whether the argument is in some way good or bad". By 'the logical evaluation of an argument', he means its evaluation "with respect to the relation between its premises and conclusion". ${ }^{6}$ Thus, in his view the logical evaluation of an argument involves at least judging whether an argument is good or bad in some way-namely, with respect to the relation between its premises and conclusion. What he argues in his reply is that the attributed (degree of) favourable relevance of an argument's premises to its conclusion "may have a bearing" on whether the argument is good or bad with respect to that relation. But to argue that the attributed (degree of) favourable relevance of an argument's premises to its conclusion may have a bearing on this 
matter is not to argue, and so is not to establish, that it does have a bearing on it. Hence Bowles's reply does not establish that it has a bearing on the logical evaluation of an argument.

Note that in his reply Bowles does not argue that the (degree of) attributed favourable relevance of an argument's premises to its conclusion is a feature of the argument itself. In his view it is not. Thus recall that by 'argument' he understands "the smallest unit of reasoning, consisting of at least one premise and exactly one conclusion" (EA:1). On this understanding, an argument does not have a component attributing to its premises (some degree of) favourable relevance to its conclusion. Such an attribution is made not by the argument itself but by the argument's associated proposition (and by the arguer if, as is usually the case, an arguer is involved ${ }^{7}$ ). If the attribution is mistaken, then the associated proposition is false: it misdescribes the relation between the argument's premises and conclusion. But how can this defect of the argument's associated proposition make the argument itself bad with respect to that relation? This question takes us to a second objection considered by Bowles.

Objection 2: Theory 6 "confuses defects of an argument with defects of a proposition about the argument's constituents. For example, according to this theory, the argument expressed in [what Bowles calls] Text 1 [viz.. "The fact that most papers are too long makes it certain that this paper is too long" (EA:1)] would be bad (invalid), because there is disagreement between the actual and attributed degrees of favorable relevance of its premise to its conclusion. But this disagreement means only that the proposition "The fact that most papers are too long makes it certain that this paper is too long', which is about that argument's premise and conclusion, is false. The truth or falsity of this proposition is irrelevant to whether the argument is valid or invalid" (EA:10). In his reply to this objection, Bowles says that "the propositions "Most papers are too long' and 'This paper is too long' constitute an argument, and one whose premise is the first of these propositions and whose conclusion is the second, only because favorable relevance to the second proposition is attributed to the first by some such associated proposition as "The fact that most papers are too long makes it certain that this paper is too long" (EA:11). He then says: "The question is whether the truth or falsity of the proposition associated with the whole argument determines whether the argument is good or bad. Theory 6's answer is affirmative"(EA:11). Bowles believes that there are two facts in favour of this answer (and so in favour of Theory 6) and one against it, and that the facts in its favour outweigh the fact against it. Against it is the fact that it requires Theory 6 to say that the argument expressed in

Text 19: The fact that all papers are too long makes it only probable that this paper is too long (EA:11)

is bad with respect to its premise-conclusion relation because the attributed degree of favourable relevance of the premise to the conclusion is less than the actual degree. To paraphrase Bowles, some will find this result intuitively correct; but others will not, for the premise entails the conclusion. Bowles himself is prepared to live with the result. The "facts" he regards as favourable to Theory 6 are, first, that of the 
six theories he examines only Theory 6 justifies "our intuitive discrimination of the good from the bad arguments" (EA:11) expressed in certain of the texts he considers, and, second, that "only Theory 6 can correctly select the weakest premise that will make" good the argument expressed in another of those texts (an argument with a missing premise) (EA:11). Let us take these points in turn.

(1) Two of the texts Bowles considers are these:

Text 2: The fact that most papers are too long makes it probable that this paper is too long (EA:1).

Text 9: The fact that most papers are too long makes the probability 0.9 that this paper is too long (EA:2).

To Bowles the argument expressed in Text 2 seems intuitively to be good, while that expressed in Text 9 seems intuitively to be bad, with respect to its premise-conclusion relation. Theory 6 justifies these intuitions, and this counts in its favour.

Consider Text 2. What argument does it express? On Bowles's understanding of 'argument', it is the following:

Premise: Most papers are too long.

Conclusion: This paper is too long.

The argument in Text 9 has the same premise and the same conclusion, but for Bowles it is nevertheless a different argument because the degree of favourable relevance to the conclusion attributed to the premise of the argument in Text 9 is different from the degree of favourable relevance to the conclusion attributed to the premise of the argument in Text $2 .^{8}$

Now the argument in Text 2 does seem intuitively to be good (with respect to its premise-conclusion relation). But why does it seem so? Three possible answers: (1) Because it is obvious that the conclusion is probable given the premise. (2) Because it is obvious that the conclusion is probable given the premise, and it is said to be made probable by (the truth of) the premise, and it seems intuitively that this is said by the argument itself. (3) Because it is obvious that the conclusion is probable given the premise, and it is said to be made probable by (the truth of) the premise, and it seems intuitively that this is said by a proposition about the argument's constituents (rather than by the argument itself). If answer (1) is correct, then the fact that the conclusion is said to be made probable by (the truth of) the premise has no bearing on our intuitive assessment of the argument; hence, if the conclusion is said by a proposition about the argument's constituents (rather than by the argument itself) to be made probable by (the truth of) the premise, that this is said by such a proposition has no bearing on our assessment, and this counts in favour of Objection 2. If answer (2) is correct, the fact that the conclusion is said to be made probable by (the truth of) the premise does have a bearing on our assessment, but it does so because (on that answer) it seems intuitively that this is said by the argument itself - not by a proposition about the argument's constituents; thus, on answer (2) we assess the argument on the basis of what it seems intuitively is said by it, not on the basis of what it seems intuitively is 
said by something else-a proposition about the argument's constituents; and this favours Objection 2. Answer (3), on the other hand, counts against Objection 2.

Next, Text 9 . It is not clear that the argument in this text seems intuitively bad with respect to its premise-conclusion relation (which is not to deny that it seems intuitively bad in some respect); after all, the argument has the same premise, and the same conclusion, as the argument in Text 2. But suppose that the argument does seem intuitively bad with respect to that relation. Why does it seem so? The answer must be: because it is obvious that, assuming the premise to be true, its truth does not make the probability of the conclusion 0.9 . But if this is the explanation, then the fact that it is said that (the truth of) the premise makes the probability of the conclusion 0.9 has a bearing on our assessment. But why does it have a bearing on our assessment? Possibly the explanation is this: it seems intuitively that it is said by the argument itself (rather than by a proposition about the argument) that (the truth of) the premise makes the probability of the conclusion 0.9 . If this is the explanation, then we assess the argument on the basis of what it seems intuitively is said by it, not on the basis of what it seems intuitively is said by something else-a proposition about the argument's constituents; and this favours Objection 2.

Bowles does not consider the possibility that the intuitive assessment he thinks we will make of the argument in Text 9 has an explanation favourable to Objection 2 . Nor does he consider the possibility that our intuitive assessment of the argument in Text 2 has such an explanation. Consequently, the first point he makes in support of Theory 6 , in his reply to Objection 2 , has less weight in that reply than he thinks.

(2) Another of the texts Bowles considers is:

Text 18: The fact that 'Evaluating Arguments: The Premise-Conclusion Relation' is a paper makes the probability 0.9 that it is too long (EA:10).

Supposing that this text "expresses an argument whose explicit premise is " "Evaluating Arguments: The Premise-Conclusion Relation" is a paper' and whose conclusion is 'It is too long'; supposing that the arguer . . has left one of his premises unexpressed; and supposing that, other things being equal, we should supply the weakest premise that will make the argument good, [then] the problem is to find the right premise" (EA:10). According to Bowles, "it seems that the premise missing from the argument in Text 18 should be ' $90 \%$ of papers are too long.' And that indeed is what Theory 6 says" (EA:10). Now the explicit components of the argument in Text 18, Bowles would say, are (just) the following:

Premise: 'Evaluating Arguments: The Premise-Conclusion Relation' is a paper.

Conclusion: It is too long.

But it seems intuitively that the premise missing from this argument should be 'All papers are too long'. That the premise missing from the argument in Text 18 should be ' $90 \%$ of papers are too long' seems intuitively to be so only if and because the attribution to the argument's explicit premise of (a degree of) favourable relevance to the 
conclusion seems intuitively to be a feature of the argument itself. Hence, either Bowles is mistaken as to what premise seems intuitively to be missing from the argument in Text 18, or he is correct but for a reason at odds with an assumption of his argument for Theory 6 (and so of his reply to Objection 2), namely that (as that objection itself assumes) the attribution to an argument's premises of (some degree of) favourable relevance to its conclusion is not a feature of the argument itself. ${ }^{9}$ In either case, the argument from intuition that Bowles makes here is unsuccessful as an argument for Theory 6 and, consequently, as a counter to Objection 2 .

In sum: Bowles contends in reply to Objection 2 that there is something to be said for, and something to be said against, the view that the truth or falsity of the proposition associated with an argument determines whether the argument is good or bad (with respect to the relation between its premises and conclusion), and that there is more to be said for this view, and so for Theory 6 , than against it. But what he says in its favour is not an adequate reply to Objection 2.

Objection 3: The proposition associated with the argument expressed in Bowles's "Text 1" is "“The fact that most papers are too long makes it certain that this paper is too long"' (EA:1). If this proposition is false, then, according to Theory 6 , the argument in Text 1 is bad with respect to the relation between its premise and conclusion. The proposition is false if it is false that most papers are too long. Thus it is false if the argument's premise is false. But if the argument's premise is false, this does not mean that the argument is bad with respect to the relation between its premise and conclusion. Thus if the proposition associated with the argument is false, this does not necessarily mean that the argument is bad with respect to that relation. But according to Theory 6 it does necessarily mean this. Accordingly, Theory 6 is mistaken in holding that whether an argument is good or bad with respect to the relation between its premises and conclusion depends entirely on whether the proposition associated with the argument is true or false.

It might be replied, however, that this objection begs the question: it assumes that if an argument has a false premise, this does not mean that the argument is bad with respect to the relation between its premises and conclusion. But, the reply continues, Theory 6 maintains precisely the opposite, if only by implication. Hence an objection to the theory begs the question if it simply assumes that an argument is not made bad with respect to the relation between its premises and conclusion by having a false premise.

I think that this is a fair reply. But I also think that it is incumbent upon a defender of Theory 6 to show that an argument is made bad with respect to the relation between its premises and conclusion by having a false premise. And this Bowles does not do. Indeed, at the beginning of his paper he distinguishes the question of whether an argument's premises are true or acceptable from the question of whether the premises are properly related to the conclusion, without suggesting that the former question is, or may be, relevant to the latter (EA:1). 
Objection 4: Even if it could be shown that an argument is made bad with respect to the relation between its premises and conclusion by having a false premise, Theory 6 would still be defective, for the following reason: it gives two criteria for when an argument is good or bad with respect to the relation between its premises and conclusion, and these criteria are not extensionally equivalent. One of the criteria is that the argument's associated proposition must be true; the other is that the attributed degree of favourable relevance of the argument's premises to its conclusion must agree with the actual degree. An argument fails the associated-proposition criterion if it has a false premise. But it might still pass the favourable-relevance criterion, for even if it has a false premise the attributed degree of favourable relevance of the premises to the conclusion might agree with the actual degree. At least this is so unless one proposition is favourably relevant to another only if the first is true. But Bowles would not say this, for in an earlier article, on propositional relevance, he proposes a theory of propositional relevance which allows that one proposition may be relevant to a second even if the first is false. ${ }^{10}$

Objection 4 is, I believe, correct. Objection 3 is also correct if (as I believe but do not here assume) an argument's having a false premise does not make it bad with respect to the relation between its premises and conclusion. But it is open to an adherent of Theory 6 to interpret the notion of an argument's associated proposition so that Objections 3 and 4 do not arise. Bowles says, recall, that "[w]ith each argument is associated a proposition that says, at the minimum, that its premises are favorably relevant to its conclusion" (EA:10-11). Objections 3 and 4 will no longer arise if to this account of an argument's associated proposition is added the clause that such a proposition does not say that the premises are true. If this addition is made, the proposition associated with the argument in Bowles's Text 1 will no longer be 'The fact that most papers are too long makes it certain that this paper is too long', but (something like) "Most papers are too long' makes certain 'This paper is too long".".2

We are left with Objections 1 and 2. Objection 1 has two parts: (1a) contrary to a presupposition of Theory 6 , the logical evaluation of an argument does not involve ascertaining whether it is good, "actually possessing features that it ought to possess" (EA:7); (1b) the logical evaluation of an argument is independent of all features that belong to the argument's context, of which one is "the (degree of) attributed favorable relevance of its premises to its conclusion" (EA:7). I did not deny that Bowles's reply to Objection 1 deals adequately with (1a), and I will assume it does. But it does not deal adequately with (1b), for Bowles does not there argue (and so does not there establish) that the attributed (degree of) favourable relevance of an argument's premises to its conclusion has a bearing on the argument's logical evaluation despite being a feature of the argument's context rather than of the argument itself. Presumably, though, Bowles would say that to refute Objection $1 \mathrm{~b}$ it is sufficient to refute Objection 2 (that the truth or falsity of a proposition about an argument's constituents is irrelevant to whether the argument is good or bad), ${ }^{12}$ and that he does refute Objection 2. But his reply to Objection 2 is, I have argued, inadequate; if so, then Bowles does not refute Objection 2 or (then) Objection $1 b$. 
It would be premature, however, to write off Theory 6 . Bowles formulates the theory in two different ways:

(1) On Theory 6, "an argument is good (valid) if the attributed and actual degrees of favorable relevance of its premises to its conclusion agree, and bad (invalid) otherwise" (EA:9).

(2) On Theory 6, "whether an argument is good or bad depends entirely on whether the proposition associated with that argument is true or false" (EA:11). ${ }^{13}$

The first version of the theory (call it Theory 6a) does not entail that associated with an argument is a proposition that attributes to the argument's premises favourable relevance to the conclusion. The second version (Theory $6 \mathrm{~b}$ ) does of course entail this, if by 'the proposition associated with an argument' is meant "'a proposition that says, at the minimum, that [the argument's] premises are favorably relevant to its conclusion" "(EA:11). ${ }^{14}$ Theory 6a is neutral on whether or not the attribution to an argument's premises of favourable relevance to the conclusion is made by the argument itself. The theory is compatible both with the view that such an attribution is made by the argument and with the view that it is not made by the argument but by a proposition associated with the argument. Now suppose that we revise Theory 6 a so that it incorporates the former view, as follows:

Theory $6 a^{\prime}$ (1): An argument is good (valid) if the degree of favourable relevance to its conclusion that it attributes to its premises agrees with the actual degree of favourable relevance of the premises to the conclusion, and is bad (invalid) otherwise.

This theory (like Theory $6 \mathrm{a}$ and Theory $6 \mathrm{~b}$ ) entails that the degree of attributed favourable relevance of an argument's premises to its conclusion is relevant to whether the argument is good or bad (with respect to the relation between its premises and conclusion). Does Objection $1 \mathrm{~b}$, or Objection 2, show that the theory is therefore mistaken? On Objection $1 \mathrm{~b}$, the logical evaluation of an argument is independent of the degree of attributed favourable relevance of its premises to its conclusion because (a) the logical evaluation of an argument is independent of all features belonging to the argument's context, and (b) the degree of attributed favourable relevance of an argument's premises to its conclusion is a feature of the argument's context, not of the argument itself. On Theory $6 \mathrm{a}^{\prime}$, (b) is false. On Objection 2, whether there is disagreement between the actual and attributed degrees of favourable relevance of an argument's premises to its conclusion is irrelevant to whether the argument is good ("valid" (EA:10)), because (c) the attribution to an argument's premises of favourable relevance to its conclusion is made by a proposition about the argument's constituents (not by the argument itself). On Theory 6a', (c) is false. On both Objection $1 \mathrm{~b}$ and Objection 2, the degree of attributed favourable relevance of an argument's premises to its conclusion is irrelevant to whether the argument is good or bad. But in support of this claim, each objection gives a reason which on Theory $6 \mathrm{a}^{\prime}$ is incorrect. Thus the 
objections fail to show that the theory is mistaken because it entails that the degree of attributed favourable relevance of an argument's premises to its conclusion is relevant to whether the argument is good or bad.

The objections fail to show this because the theory assumes that an argument itself attributes to its premises (some degree of) favourable relevance to its conclusion. But how does it do this? Compatibly with Theory 6a' we may answer: by means of a proposition which says that the premises are (to some degree) favourably relevant to the conclusion. On this view, arguments do not consist (as they do for Bowles) just of premises and conclusions, but have a third element, which is a proposition about the other two. Compare James Freeman:

Arguments ... involve these three factors: premises, conclusions, and a claim that the premises support the conclusions. ${ }^{15}$

Of course it could be held that these factors are 'involved' in arguments in different ways: that premises and conclusions are constituent features of arguments, while a claim that the premises support the conclusion is associated with (not an element of) an argument. But in the text from which I have quoted, Freeman gives no sign of holding this view; he appears to think, rather, that the three factors he says are involved in arguments are, all of them, constituent features of arguments. Monroe Beardsley also holds a 'three-factor' view of arguments:

[1]n logic, an argument is a special kind of discourse, embodying the claim [italics added] that one or more specific statements [conclusions] ought to be accepted as true, or probably true, just because certain other statements [premises] are true. ${ }^{16}$

Thus for Beardsley, as apparently for Freeman, arguments have three elements: premises, conclusions, and a claim about the premises and conclusions.

Now it is clear that 'Theory 6a' presupposes either this same view of arguments, or a view on which arguments include some third element over and above premises and conclusions, but one that is not a claim. I am going to suppose that for that theory the third element is a proposition-one attributing to the argument's premises (some degree of) favourable relevance to the conclusion; I will call such a proposition a relevance proposition. For Theory $6 \mathrm{a}^{\prime}$, then, the simplest argument will consist of one premise, one conclusion, and one relevance proposition. The generic form of such an argument can be exhibited as follows, where ' $p$ ' and ' $q$ ' stand for propositions:

Form A: (1) $p$

(2) $q$

(3) ' $p$ ' is favourably relevant to ' $q$ '. ${ }^{18}$

An argument of Form $A$ can be expressed otherwise than in a text of that form: it can be expressed in a text of the form ' $p$; therefore, $q$ '. This is because a text of that form connects ' $p$ ' to ' $q$ ' by means of an illative ('therefore'), and, as Bowles says, "illatives are expressions of attributed favorable relevance" (EA:7). Thus a text of that form expresses what is said by the relevance proposition of an argument of Form A (namely, 
that ' $p$ ' is favourably relevant to ' $q$ '), as well as the premise (' $p$ ') and the conclusion (' $q$ ') of an argument of that form. (Bowles, for his part, would say that a text of that form expresses the least that is said by the proposition associated with an argument whose premise is ' $p$ ' and whose conclusion is ' $q$ ', namely that ' $p$ ' is favourably relevant to ' $q$ ', as well as the argument's premise and conclusion.)

Theory $6 \mathrm{a}^{\prime}, \mathrm{I}$ am supposing, assumes that every argument includes a relevance proposition. Let us assume that this is true. Let us further assume that (in addition to its relevance proposition) an argument has "at least one premise and exactly one conclusion" (EA:1). We can then make the following three points. (1) Like a proposition of the sort that in Bowles's view is associated with an argument, an argument's relevance proposition says, "at the minimum, that [the argument's] premises are favorably relevant to its conclusion" (EA:11). (2) An argument's relevance proposition, again like a Bowlesian associated proposition, can be "more detailed, saying, for example, either that the premises are conclusively favorably relevant to the conclusion or that they are to some degree inconclusively favorably relevant to it" (EA:11). (3) An argument's relevance proposition is true just in case the degree of favourable relevance to the conclusion that it attributes to the premises agrees with the actual degree of favourable relevance of the premises to the conclusion.

Given this last point we can state Theory $6 a^{\prime}$ more succinctly, as follows:

Theory $6 a^{\prime}(2)$ : an argument is good (valid) just in case its relevance proposition is true.

According to this theory, then, the truth of an argument's relevance proposition is both necessary and sufficient for the argument to be good-good, that is, with respect to the relation between its premises and conclusion. In a moment I will present an argument for the view that the truth of an argument's relevance proposition is necessary for the argument to be good with respect to that relation. First, however, let us return briefly to Bowles. As we have seen, one of the texts he considers is:

Text 1: The fact that most papers are too long makes it certain that this paper is too long (EA:1).

To Bowles, it seems intuitively that the argument in this text is bad with respect to its premise-conclusion relation; it is, he believes, an argument "involving a logical error" (EA:6). The error is that of mistaking the degree to which the argument's premise is favourably relevant to the conclusion. Hence it is a mistake about the (logical) relation between the premise and the conclusion. If an argument is one "involving" such a mistake, this (for Bowles) makes it bad with respect to the relation between its premise(s) and conclusion. This idea, slightly altered, figures in premise (2) of the following argument:

(1) If an argument's relevance proposition is false, the argument contains a logical error concerning the relation between its premises and conclusion. 
(2) If an argument contains such an error, it is a bad argument with respect to that relation.

(3) Thus, if an argument's relevance proposition is false, the argument is bad with respect to that relation.

(4) Thus, if an argument is good with respect to that relation, its relevance proposition is true.

(5) Thus, the truth of an argument's relevance proposition is necessary for the argument to be good with respect to the relation between its premises and conclusion.

If premise (2) of this argument is correct, then, if an argument contains a mistake about the relation between its premises and conclusion, this has the consequence that that relation is itself defective - that the argument's premises are not properly related to its conclusion. But this is precisely what a case for Theory 6a' must establish. And it is not established by the present argument, for the argument provides no support for premise (2). Rather, it begs the question.

A case for Theory $6 \mathrm{a}^{\prime}$ will face the same difficulty that a case for Theory $6 \mathrm{~b}$ would face: that of showing that the truth or falsity of a proposition about an argument's premises and conclusion is relevant to whether the argument is good or bad with respect to the relation between its premises and conclusion. It makes no difference whether the proposition is associated with the argument, as it is for Theory $6 \mathrm{~b}$, or a constituent of the argument, as I am supposing it is for Theory 6a'. The same difficulty arises in either case. And 1 have no solution for it. Accordingly, 1 will make no further attempt to defend Theory $6 a$ '.

There is, however, a consequence of that theory that 1 wish to consider, namely that, in the case of any argument, the truth or falsity of its relevance proposition is relevant to its evaluation. This view presupposes that in the case of any argument there is some proposition which is that argument's relevance proposition. We are, of course, assuming that this is so, for we are assuming that an argument has a relevance proposition as one of its constituents. Now an argument is defective if one of its constituents, other than its conclusion, is defective. (I assume here, and below, that it is possible for an argument with a false, and so defective, conclusion to be a good argument; and, to simplify discussion, I continue to assume, as I do below, that an argument has exactly one conclusion.) If then, as we are assuming, an argument has a relevance proposition as one of its constituents, then, since that proposition is not the argument's conclusion, if it is false, and so defective, the argument is not in all respects good. Hence, if an argument has a relevance proposition as one of its constituents, the truth or falsity of that proposition is relevant to the argument's evaluation.

I will continue to assume that an argument does indeed have a relevance proposition as one of its constituents. On this assumption, then, if an argument's relevance proposition is true, this is a virtue of the argument. Moreover, it is one that the argument must have if the reasoning that is the argument, or that is in the argument, is good reasoning. 
To elaborate: there are different views about the relation between argument and reasoning. On one view, an argument is something in which there is reasoning. This view is held by Michael Scriven, as is evident from the following passage:

We often talk about an argument being 'logically sound' in order to contrast it with its being 'factually sound'. To say that the argument is logically sound is to say that the reasoning in it is sound, that the inference(s) from the premises to the conclusion(s) is/are sound, without saying anything about whether the premises are themselves sound..$^{18}$

On a different view, reasoning is not something (that goes on) in an argument; rather, reasoning is an argument, and an argument is (a piece of) reasoning. This view is held by Bowles. ${ }^{19}$

If an argument is reasoning (not something in which there is reasoning), then, since (as I am assuming) an argument has a relevance proposition as one of its constituents, such a proposition is an element of the reasoning that is an argument; but it is not the conclusion of that reasoning. If, then, it is false (and so defective), then that reasoning is defective. Thus, if an argument is reasoning (not something in which there is reasoning), it is good reasoning only if its relevance proposition is true..$^{20}$ (But the truth of an argument's relevance proposition would not be sufficient for the argument to be good reasoning; the reasoning that is the argument is good reasoning only if the argument satisfies further conditions, including, for example, that of having true or acceptable premises. ${ }^{21}$ )

I will now assume that an argument is, rather, something in which there is reasoning, and argue that an argument is well-reasoned (i.e., that its reasoning is good) only if its relevance proposition is true. (I will not, however, argue that the truth of an argument's relevance proposition is sufficient for the argument to be well-reasoned. It is not, if (for example) begging the question is an error in reasoning, for the relevance proposition of a question-begging argument may be true. ${ }^{22}$ )

My argument is the following:

(1) If an argument's relevance proposition is false, the argument contains a logical error concerning the (logical) relation between its premises and conclusion.

(2) If an argument contains such an error, it is not well-reasoned.

(3) Thus, if an argument's relevance proposition is false, the argument is not well-reasoned.

(4) Thus, an argument is well-reasoned only if its relevance proposition is true.

A defense of premises (1) and (2). Premise 1: An argument's relevance proposition says that the argument's premises stand in a certain logical relation to the conclusion, viz., that of being (to some degree) favourably relevant to the conclusion. The proposition is false just in case the logical relation between the argument's premises and conclusion is other than as the proposition says it is. Thus, if the proposition is false, it is mistaken as to the nature of that logical relation. Such a mistake is a logical 
error. If then an argument's relevance proposition is false, then, since (as I am assuming) that proposition is a constituent of the argument, the argument contains a logical error concerning the (logical) relation between its premises and conclusion. Thus, premise (1) is true. Premise $2:$ In the set of propositions that constitutes an argument, the relevance proposition corresponds to the arguer's inference, if an arguer is involved. That proposition is (the locus of) the reasoning in the argument. Now if an argument contains a logical error conceming the (logical) relation between its premises and conclusion, the error is located in its relevance proposition, hence in that component of the argument which is (the locus of) its reasoning, and so the argument is not well-reasoned. Thus, premise (2) is true. (On a different view, an argument's relevance proposition is one component of the reasoning in an argument; another component is the argument's premises. But even if this is so, premise (2) is still true. For if an argument's relevance proposition is mistaken as to the nature of the (logical) relation between the argument's premises and conclusion, this logical error is still located in the reasoning in the argument, and so the argument is not well-reasoned.)

I have now argued that if an argument is reasoning (not something in which there is reasoning), it is good reasoning only if its relevance proposition is true; and, assuming that an argument is, rather, something in which there is reasoning, that only if its relevance proposition is true is it well-reasoned. Thus, a necessary condition of good argumentative reasoning is the truth of an argument's relevance proposition. Since an argument's relevance proposition attributes to the argument's premises (some degree of favourable relevance to the conclusion, we may call this necessary condition of good argumentative reasoning the attributed-relevance condition.

Now to determine whether an argument satisfies this condition, it must be decided whether what is said by the argument's relevance proposition is something "more detailed" (EA:11) than that the argument's premises are favourably relevant to its conclusion-for example, that they are conclusively, or that they are (to some degree) inconclusively, favourably relevant to it. It must also be decided whether the premises are favourably relevant to the conclusion (either conclusively or, to some degree, inconclusively). Accordingly, before the condition can be used in the evaluation of an argument, it must be supplemented - by guidelines for making the first of these decisions, and, for the purpose of making the second of them, by some view of when one proposition is, and when it is not, favourably relevant to another. Neither of these topics, however, will I take up here. ${ }^{23}$

I have assumed that an argument has a relevance proposition as one of its constituents. I now want to ask whether this assumption is correct. Suppose it is correct. Then an argument has an element attributing to its premises favourable relevance to its conclusion, and this element of an argument is a proposition. Now it is clear that one proposition is the premise of an argument, another the conclusion, only because favourable relevance to the latter is attributed to the former (cf. EA:11). The attribution is made by someone or by something. It is made by someone, namely the arguer, if an arguer is involved. Is it also made by something? I am going to assume that it is; I am 
also going to assume that the thing by which it is made is a proposition. On this assumption, then, an argument must have somehow related to it a proposition attributing to its premises favourable relevance to its conclusion, or it would not be an argument. Such a proposition is appropriately called the argument's relevance proposition. But the relation between an argument's relevance proposition and the argument itself need not be thought of as one of part to whole; it could be thought of instead as one of association. In other words, an argument's relevance proposition need not be thought of as something the argument has within it, that is, as an element of the argument; it could be thought of instead as something the argument has associated with it. Accordingly, assuming that in the case of each argument there is a proposition, called the argument's relevance proposition, which attributes to the argument's premises favourable relevance to its conclusion, and which is either an element of the argument or is associated with it, let us ask which it is: is it an element of the argument, or is it associated with the argument? Or is it not the case that one of these views is correct, the other incorrect, but that they are simply different views about the relation between an argument and its relevance proposition? I am inclined to think that one of the views is correct, and that it is the former view-that an argument's relevance proposition is an element of the argument. In support of this view, I offer the following argument. As Bowles and Gilbert have written " $[w]$ here favorable relevance to one proposition is not at least attributed to another, there is no reasoning from the second to the first". ${ }^{24}$ If there is reasoning from the second to the first, either it consists entirely in the attribution to the second of favourable relevance to the first, or it consists partly in such an attribution. Hence it consists at least partly in such an attribution. Now if an argument is reasoning (not something in which there is reasoning), the reasoning that is an argument is reasoning from the argument's premises to its conclusion. The premises and the conclusion are parts of the reasoning, if the reasoning is the argument; but if reasoning from one proposition to another consists at least partly in the attribution to the former of favourable relevance to the latter, then, if an argument is reasoning, a further part of the reasoning that is an argument is an attribution to the argument's premises of favourable relevance to its conclusion, in which case the argument has an element that makes such an attribution, and this is the argument's relevance proposition. If, on the other hand, an argument is something in which there is reasoning, an attribution to an argument's premises of favourable relevance to its conclusion is, or is part of, the reasoning that is in the argument, in which case the argument has an element that makes such an attribution, and this is the argument's relevance proposition. Now either an argument is reasoning or it is something in which there is reasoning. In either case, an argument's relevance proposition is one of its elements. Hence an argument's relevance proposition is an element of the argument.

This completes my response to the question of whether it is true (as I earlier assumed it to be) that an argument has a relevance proposition as one of its constituents. I have assumed that in the case of each argument there is such a proposition somehow related to it, and have argued that the proposition is an element of the argu- 
ment; on that assumption, then, an argument does have a relevance proposition as one of its constituents.

Final remarks:

(1) Suppose that an argument is something in which there is reasoning. Then (as I have argued) the truth of its relevance proposition is necessary for it to be well-reasoned. But suppose that, contrary to Theory $6 \mathrm{a}^{\prime}$, the truth of its relevance proposition is not necessary for it to be good with respect to the relation between its premises and conclusion. Then it is not the case that for an argument to be well-reasoned just is for it to be good with respect to that relation. But even if this is so, there is a connection between an argument's being good with respect to that relation and its being wellreasoned. For there is a condition that is necessary both for an argument to be good with respect to that relation and for it to be well-reasoned, namely that its premises be favourably relevant to its conclusion. This condition is obviously necessary for an argument to be good with respect to the relation between its premises and conclusion-that is, for its premises to be properly related to its conclusion. And the condition is also necessary for an argument to be well-reasoned, given that an argument is well reasoned only if its relevance proposition is true; for an argument's relevance proposition is true only if the argument's premises are favourably relevant to its conclusion. Now suppose instead that an argument is reasoning (not something in which there is reasoning). Then the same condition (viz., that an argument's premises be favourably relevant to its conclusion) is for the same reasons necessary both for the argument to be good with respect to the relation between its premises and conclusion, and for it to be good reasoning.

(2) Theory 6 (in either version) and Theory 6a' have the consequence that the degree of attributed favourable relevance of an argument's premises to its conclusion is relevant to its evaluation. And this consequence is correct, at least on the assumption (for which I have argued) that an argument has a relevance proposition as one of its constituents. For, as I have on that assumption also argued, an argument is good reasoning, or is well-reasoned, enly if its relevance proposition is true, and its relevance proposition is true just in case the attributed and actual degrees of favourable relevance of its premises to its conclusion agree.

\section{Notes}

${ }^{1}$ This is an extensively revised version of a paper (Allen 1995) that I read at Conference 95 on Critical Thinking and Informal Logic, George Mason University, June 1995. I am most grateful to George Bowles for providing me with detailed and very helpful written comments on that paper.

2 By 'in an argument', Bowles does not here mean 'within an argument'. Rather, he means (something like) 'in the case of an argument'. (Relevant to this point is my comment later in the text on Bowles's reply to Objection I to Theory 6.)

${ }^{3}$ Bowles attributes Theory 6 to me, on the basis of Allen 1988.1 comment on this attribution in Note 14. 
${ }^{4}$ In the summary I later give of Bowles's reply to Objection 2 to Theory 6 , I report what he thinks is to be said for and against the theory.

${ }^{5}$ The first of these objections is one of several that Bowles raises against Theory 5 , and to which he thinks Theory 6 is also subject; he designates it Objection 1 (to Theory 5); I also designate it Objection 1 (but to Theory 6 ). The second of the objections is one he raises just against Theory 6; he does not number that objection; I do-as Objection 2.

${ }^{6} \mathrm{Cf}$. EA: 1 , paragraph 1 .

${ }^{7}$ In Bowles's view, an argument need not involve an arguer: "an argument might be expressed independently of any rational agency - e.g., by unusual erosion on a hillside or an improbable arrangement of colored pebbles on a beach" (Bowles 1989:13); an argument expressed independently of any rational agency does not involve an arguer, if, as Bowles holds, an arguer is a "person or other rational being" (ibid.).

8 "One argument is differentiated from another by at least these three things: premises, conclusions, or degrees of attributed favorable relevance" (EA:2).

${ }^{9}$ The relevant part of Bowles's argument can be reconstructed as follows:

(1) Theory 6 selects ' $90 \%$ of papers are too long' as the premise missing from the argument in Text 18.

(2) That selection seems intuitively correct.

(3) Thus, the fact that Theory 6 makes that selection counts in favour of the view that

(a) the truth or falsity of the proposition associated with an argument determines whether the argument is good or bad with respect to the relation between its premises and conclusion,

and so it counts in favour of Theory 6 itself.

Plainly this argument assumes that Theory 6 affirms (a). Underlying this assumption is the further assumption that the attribution to an argument's premises of (some degree of favourable relevance to the conclusion is not a feature of the argument itself (but is made by the argument's associated proposition); for if it were a feature of the argument itself, there would be no need to introduce the notion of an argument's associated proposition in connection with Theory 6 , hence no need to argue for that theory via the assumption that it affirms (a). (Further, there would be no need to reply to Objection 2, as Bowles does, by defending (a), for the objection could then be refuted on the ground that it is mistaken in its assumption that the attribution to an argument's premises of (some degree of) favourable relevance to the conclusion is not a feature of the argument itself.)

${ }^{10}$ Bowles 1990.

"In his comments on Allen 1995, Bowles granted Objections 3 and 4, and said that he should not have used, as an example of an associated proposition, 'The fact that most papers are too long makes it certain that this paper is too long', but (something like) "'Most papers are too long' makes certain "This paper is too long",

${ }^{12}$ If Objection 2 is incorrect, then the truth or falsity of an argument's associated proposition is relevant to the argument's evaluation; if so, then the (degree of) attributed favourable relevance of the argument's premises to its conclusion is relevant to its evaluation. But according to Objection Ib, such favourable relevance is a feature of an argument's context; if so, then, if Objection Ib is correct, such favourable relevance is not relevant to an argument's evaluation. If such favourable relevance is relevant to an argument's evaluation, then Objection Ib is not correct. Thus, if Objection 2 is incorrect, so is Objection $\mathrm{Ib}$. Hence, to refute Objection $1 \mathrm{~b}$ it would be sufficient to refute Objection 2.

${ }^{13}$ When Bowles speaks in EA of an argument's being good or bad, he always intends, even when he omits, "the qualification "with respect to the relation between its premises and conclusion" (EA:1). 
${ }^{14}$ In Note 4, I said that Bowles attributes Theory 6 to me, on the basis of Allen 1988. I have now distinguished two versions of that theory-Theory $6 \mathrm{a}$ and Theory $6 \mathrm{~b}$. Between Theory $6 \mathrm{a}$ and the theory I defend in Allen 1988 there are verbal differences: I do not use the expression 'attributed degree of favourable relevance', or the expression 'actual degree of favourable relevance', to state the theory. Between Theory $6 \mathrm{~b}$ and the theory I defend in Allen 1988 there is also a verbal difference: I do not use the expression 'the proposition associated with an argument' to state the theory. This difference is connected to a significant substantive difference between the two theories: the theory I defend does not assume that the attribution to an argument's premises of favourable relevance to the conclusion is not a feature of the argument itself; on the contrary, that theory would say that such an attribution is made in the argument, by means of what in Allen 1988 I call an inference claim.

${ }^{15}$ Freeman 1993:35.

${ }^{16}$ Beardsley 1975:12.

${ }^{17}$ Given (3), it is not necessary (but would be redundant) to label (1) 'premise' and (2) 'conclusion'. (Cf. EA:1 1, paragraph 1.)

${ }^{18}$ Scriven 1976:38. Scriven also speaks of "the reasoning step in an argument", and says that "[t]he argument itself, stated in full, consists in first asserting that the premises are true, and then asserting the reasoning step or the inference- that is, asserting that the premises imply a particular conclusion- and hence, in light of this, affirming the conclusion" (Scriven 1976:46).

"As we have seen, Bowles says that by 'argument' he understands "the smallest unit of reasoning, consisting of at least one premise and exactly one conclusion" (EA:1). And in his comments on Allen 1995, he wrote that "reasoning is an argument". In those comments he also said that talk of an argument's being well-reasoned makes him uncomfortable because it suggests (what in his judgment is false) that reasoning is something that goes on in an argument. I have this view of his in mind when, on the assumption that an argument is something in which there is reasoning, I later speak of an argument's being (or not being) well reasoned

${ }^{20}$ If an argument's relevance proposition is an element (other than the conclusion) of the reasoning that is the argument, it is unmysterious that if it is false, and so defective, its being thus defective should make that reasoning defective. By contrast, an argument's relevance proposition is not a relation, or part of a relation, between the argument's premises and conclusion; a fortiori, it is not identical to, or part of, the logical relation between the argument's premises and conclusion; hence, it is natural to question whether, if that proposition is false, and so defective, its being thus defective makes that relation (and so the argument with respect to that relation) defective.

${ }^{21}$ Robert $\mathrm{C}$. Pinto, J. Anthony Blair, and Katharine E. Parr take the view that good reasoning requires acceptable premises, in Pinto, Blair and Parr 1993 at p. 95.

${ }^{22}$ The relevance proposition of a question-begging argument is true if it says (just) that the argument's premises are favourably relevant, or that they are conclusively favourably relevant, to the conclusion.

${ }^{23}$ Material relevant to the former topic is to be found in Allen 1988. (In that paper I use the term 'inference claim' for what 1 here call an argument's relevance proposition.) See also Bowles and Gilbert 1993. In connection with the latter topic, see Bowles 1990. (Bowles holds that one proposition is favourably relevant to another just in case the second is probable or certain given the first.)

${ }^{24}$ Bowles and Gilbert 1993:249. 


\section{References}

Allen, Derek, "Inferential Soundness", Informal Logic, Vol. x, No. 2 (Spring 1988), 57-65.

Allen, Derek, "When Is An Argument Well Reasoned?", read at Conference 95 on Critical Thinking and Informal Logic, George Mason University, June 1995. (Unpublished.)

Beardsley, Monroe, Thinking Straight, Prentice-Hall, Inc., Englewood Cliffs, New Jersey, 1975. Bowles, George, "Favorable Relevance and Arguments", Informal Logic, Vol. xi, No. 1 (Winter 1989), 11-17.

Bowles, George, "Propositional Relevance", Informal Logic, Vol. xii, No. 2 (Spring 1990), 6577.

Bowles, George, "Evaluating Arguments: The Premise-Conclusion Relation", Informal Logic, Vol, xiii, No. 1 (Winter 1991), 1-20.

Bowles, George and Gilbert, Thomas E., "The Probabilistic Import of Illatives", Argumentation 7:247-262, 1993.

Freeman, James B., Thinking Logically: Basic Concepts for Reasoning, Prentice-Hall, Inc., Englewood Cliffs, New Jersey, 1993.

Pinto, Robert C., Blair, J. Anthony, and Parr, Katharine E., Reasoning, Prentice-Hall, Inc., Scarborough, Ontario, 1993.

Scriven, Michael, Reasoning, McGraw-Hill Book Company, New York, 1976. 